\title{
Hautsymptome bei COVID-19
}

COVID-19 wird in erster Linie als Atemwegserkrankung betrachtet. SARS-CoV-2 befällt aber auch viele andere Organe. Das Virus kann zu Herzproblemen, zu Nierenversagen und zu Hyperglykämie führen. Es kann die Verdauung angreifen und die Leberfunktion beeinträchtigen und es kann neurologische Symptome hervorrufen. An der Haut zeigt sich COVID-19 überraschend selten und unspezifisch: Eine ganze Reihe von sehr unterschiedlichen Hauterscheinungen wurden bisher mit dem Virus in Verbindung gebracht. Prof. Dr. Harald Kittler, Dermatologe an der MedUni Wien, erklärt wie die vielen Berichte zu COVID-19 und Hautsymptomen zu bewerten sind und was sie für die Praxis bedeuten.

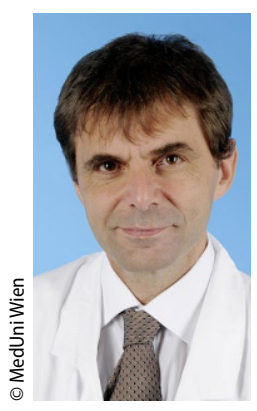

$\Delta$ Prof. Dr. Harald Kittler
Warum befällt SARS-CoV-2 so viele Organe?

Kittler: Die Eintrittspforte von SARS-CoV-2 ist der ACE-2-Rezeptor, der nicht nur in der Lunge sitzt, sondern fast im ganzen Körper. SARS-CoV-2 kann also an verschiedenen Stellen in den Körper eindringen. Außerdem werden durch die Infektion verschiedene Botenstoffe mit systemischer Wirkung freigesetzt. Es ist mitunter schwer zu unterscheiden, ob wir mehr unter dem Virus oder unter unseren Abwehrmechanismen leiden.

Gibt es auch an der Haut ACE-2Rezeptoren?

Kittler: Sehr wahrscheinlich verfügen auch die Gefäße, die die Haut ernähren, über ACE-2-Rezeptoren. Möglicherweise tragen auch die Drüsenstrukturen der Haut ACE-2-Rezeptoren, allerdings nicht in derselben Dichte, in der ACE-2-Rezeptoren in anderen Organen vorkommen. In der Epidermis selbst finden sich vermutlich keine ACE-2-Rezeptoren.

Es gibt viele Fallberichte zu Hauterscheinungen, die mit COVID-19 in Zusammenhang gebracht werden. Sind alle diese Berichte gleich relevant?

Kittler: Die Pandemie sorgt für viel Aufregung. Bei jeder Nachricht, die im Zusammenhang mit COVID-19 steht, steigt die Aufmerksamkeitsschwelle. Das hat dazu geführt, dass es auch viele unzusammenhängende Berichte und schnell abgewickelte Publikationen gibt. Bei dieser großen Fülle an Information ist es schwierig, die Relevanz zu werten.

Viele der Berichte von Hauterscheinungen im Zusammenhang mit COVID-19 sind möglicherweise unspezifisch. Wenn eine Erkrankung pandemisch auftritt, werden auch zufällige Zusammenhänge berichtet und es ist schwierig, die zufälligen Verbindungen von den kausalen Verbindungen zu unterscheiden. Es scheint so zu sein, dass es wenige kausale Zusammenhänge zwischen COVID-19 und Hauterscheinungen gibt, aber einige wenige doch. Und diese wenigen aus der Fülle an Information herauszufiltern, ist herausfordernd und noch nicht abgeschlossen. Die meisten Berichte zu SARS-CoV-2 und Hautmanifestationen sind Fallberichte, zu denen es noch keine systematischen Untersuchungen gibt.

Review-Papers sind sicher eine bessere Informationsquelle zum Thema. Was auffallend ist: Während der ersten COVID-19-Welle wurde in der wissenschaftlichen Literatur viel mehr über Hauterscheinungen berichtet als während der zweiten und dritten Welle. Die wissenschaftliche Berichterstattung zu Hautsymptomen bei COVID-19 ist deutlich abgeflaut.

Wie häufig zeigt sich COVID-19 an der Haut?
Kittler: Die ersten Berichte aus Italien, die Hauterscheinungen bei COVID-19 erwähnen, sprechen von Hautsymptomen bei $20 \%$ der infizierten Personen. Das ist sicher ein viel zu hoher Prozentsatz. Hier ist Selektions-Bias im Spiel und man muss sich ansehen, wie solche Prozentzahlen zustande kommen: Es wurden alle Patienten mit Hauterscheinungen und COVID-19 zusammengefasst und alle COVID-19-Infizierten. Allerdings war die Gesamtzahl der Infizierten in der ersten Infektionswelle nicht genau zu erfassen.

Auf der anderen Seite sprechen erste Berichte aus China über nahezu $0 \%$ Hautsymptome bei COVID-19 und dieser Prozentsatz ist vermutlich zu niedrig. Die Wahrheit ist wahrscheinlich irgendwo dazwischen: Ich würde schätzen, dass $1 \%$ oder weniger der Patienten mit COVID-19 Hautsymptome zeigen.

Von welchen Hautsymptomen wird hier berichtet?

Kittler: Interessant ist, dass im Zusammenhang mit COVID-19 eine Vielzahl von Hautsymptomen beschrieben wurde - also nicht nur ein Ausschlag, wie wir das von anderen viralen Infektionserkrankungen gewohnt sind. Bei Varicellen etwa zeigt sich ein typisches Exanthem mit Bläschen. Auch bei Masern oder Röteln gibt es typische Erscheinungsbilder, der Ausschlag ist immer gleich. Das ist bei COVID-19 nicht der Fall. 
Es gibt Berichte über vesikulöse Ausschläge, urtikarielle Ausschläge und Schießscheiben-artige Läsionen wie sie auch nach einer Herpes Virus-Infektion auftreten können. Auch unspezifische Arzneimittelexanthem-artige Ausschläge werden berichtet sowie Petechien und nekrotische Läsionen.

Am bekanntesten sind wahrscheinlich die COVID-Zehen, die aussehen wie Erfrierungen, Kälteflecken oder Frostbeulen an den Zehen oder Fingern und die vor allem bei jüngeren Patienten im späten Verlauf der COVID-19-Infektion auftreten können.

Sie sehen an der breiten Palette der genannten Symptome, dass es sehr wahrscheinlich keinen COVID-19-spezifischen Hautausschlag gibt. Die berichteten Hauterscheinungen sind ein Mix aus unspezifischen und etwas spezifischeren Symptomen an der Haut. Mit unspezifischen Symptomen meine ich, dass viele Infektionen zum Beispiel Nesselausschläge triggern können. So können Sie etwa nach einer Erkältung einen Nesselausschlag bekommen, das ist gar nicht so selten. Auch COVID-19 kann zu solchen para-infektiösen nicht-spezifischen Phänomenen führen. Bei Varicellen kann der Hautausschlag zur Diagnose führen, bei COVID-19 kann die Diagnose sicher nicht aufgrund von Hauterscheinungen gestellt werden.

Gibt es einen Zusammenhang zwischen Hautsymptomen mit dem Schweregrad der COVID-19-Erkrankung?

Kittler: Eher nicht - die Ausschläge treten in unterschiedlichen Stadien der Erkrankung auf. Vesikulöse und urtikarielle Ausschläge sehen wir eher zu Beginn der Erkrankung, die so genannten COVID-Zehen zeigen sich eher später im Verlauf. Es gibt Hautsymptome, die Ausdruck eines schweren Krankheitsver- laufs, einer Sepsis oder eines Multiorganversagens, sind. Diese Veränderungen treten natürlich dann auf, wenn es dem Patienten sehr schlecht geht, aber sie sind nicht COVID-19-spezifisch.

Haben manche Patienten - etwa unterBiologika-Therapie-ein höheres Risiko Hautsymptome zu entwickeln? Kittler: Patienten, die immunsupprimiert sind, zählen zu den Risikogruppen für einen schwereren Krankheitsverlauf. Ganz klar sind die Zusammenhänge bei den einzelnen Gruppen von Immunsuppressiva aber nicht. Es könnte zum Beispiel auch sein, dass Patienten Biologika aufgrund einer Hauterkrankung erhalten und dann schon wegen Ihrer Grunderkrankung eher zu Hautsymptomen neigen. Wer etwa für seine Psoriasis ein Biologikum bekommt, kann auch bei einer COVID-Infektion PsoriasisLäsionen zeigen. Diese sind aber nicht unbedingt ursächlich mit der COVID-Infektion verknüpft.

Was bedeuten Hautsymptome für die Praxis?

Kittler: Die Antwort auf diese Frage ist nicht einfach. Viele Patienten mit COVID-Zehen etwa sind asymptomatisch in Bezug auf COVID-19 oder haben nicht einmal Antikörper für SARS-CoV-2. Natürlich möchte man COVID-19-Patienten erkennen, um sie unter Quarantäne zu stellen. Aufgrund von Hauterscheinungen ist das aber nicht möglich.

In Österreich sind wir Testweltmeister. Wenn ich also, wie in Österreich, eine Teststrategie verfolge, bei der möglichst alle möglichst häufig getestet werden sollen, dann werde ich auch jemanden mit Urtikaria testen, weil die Testschwelle ohnehin niedrig ist. Wenn sich die Teststrategie ändert und die Schwelle für Testungen, wie etwa in Deutschland, höher liegt, würde ich sagen: Hautsymptome zählen nicht unbedingt zu jenen Symptomen, die Anlass geben jemanden als Risikoperson zu betrachten.

Nachdem Sie sich in den letzten Monaten mit dem Thema COVID-19 und Haut beschäftigt haben - was hat Sie überrascht?

Kittler: Eigentlich sollte uns nichts mehr überraschen, was mit COVID-19 zusammenhängt. „Optimismus ist Pflicht", um einen österreichischen Philosophen zu paraphrasieren. Positiv überrascht hat mich, dass es innerhalb eines Jahres möglich war, so viele Impfstoffe mit hoher Wirkung herzustellen. In einer Situation, die sicher nicht leicht war, wurde zumindest der Versuch einer rationalen Lösung eines weltweiten Gesundheitsproblems, das wir seit der Spanischen Grippe nicht mehr gehabt haben, gestartet. Vor 100 Jahren war eine Impftherapie nicht möglich. Wir sollten eigentlich froh sein, dass wir in einer Zeit leben, wo innerhalb eines Jahres Impfstoffe zur Verfügung gestellt werden können. Das ist eine tolle Leistung, auf die wir als Menschen stolz sein sollten.

\section{Das Gespräch führte \\ Mag. Tanja Fabsits}

Das Interview gibt es auch zu hören - im "Hörgang", dem Podcast von SpringerMedizin.at, unter anderem unter https://bit.ly/ 2RE05hi oder über iTunes, Spotify und Podigee.

Hinweis des Verlags. Der Verlag bleibt in Hinblick auf geografische Zuordnungen und Gebietsbezeichnungen in veröffentlichten Karten und Institutsadressen neutral.

hautnah $2021 \cdot 20: 84-85$

https://doi.org/10.1007/s12326-02100448-4

(c) Springer-Verlag GmbH Austria, ein Teil von Springer Nature 2021 